Article

\title{
Genome-Wide Association Analysis Identified BMPR1A as a Novel Candidate Gene Affecting the Number of Thoracic Vertebrae in a Large White $\times$ Minzhu Intercross Pig Population
}

\author{
Qian Liu ${ }^{\dagger}$, Jingwei Yue ${ }^{\dagger}$, Naiqi Niu, Xin Liu, Hua Yan, Fuping Zhao $\odot$, Xinhua Hou, \\ Hongmei Gao, Lijun Shi, Lixian Wang *, Ligang Wang and Longchao Zhang * \\ Institute of Animal Science, Chinese Academy of Agricultural Sciences, Beijing 100193, China; \\ liuqian521818@163.com (Q.L.); qgww007@126.com (J.Y.); 18734450897@163.com (N.N.); \\ firstliuxin@163.com (X.L.); zcyyh@126.com (H.Y.); zhaofuping@caas.cn (F.Z.); 7hxh73@163.com (X.H.); \\ gaohongmei_123@126.com (H.G.); shilijun01@caas.cn (L.S.); ligwang@126.com (L.W.) \\ * Correspondence: wanglixian@caas.cn (L.W.); zhanglongchao@caas.cn (L.Z.); Tel.: +86-010-6281-8771 (L.Z.) \\ + These authors contribute equally to this work.
}

Received: 9 October 2020; Accepted: 6 November 2020; Published: 22 November 2020

Simple Summary: The number of thoracic vertebrae (NTV) and number of vertebrae (NV) varies among pig breeds with a high correlation of about 0.8 . It is important to discover variants associated with the NTV by considering the effect of the NV in pig. The results suggest that regulation variants on SSC7 might play crucial roles in the NTV and the FOS on SSC7 should be further studied as a critical candidate gene. In addition, BMPR1A was identified as a novel candidate gene affecting the NTV in pigs.

\begin{abstract}
The number of vertebrae (NV), especially the number of thoracic vertebrae (NTV), varies among pig breeds. The NTV is controlled by vertebral segmentation and the number of somites during embryonic development. Although there is a high correlation between the NTV and NV, studies on a fixed NV have mainly considered the absolute numbers of thoracic vertebrae instead of vertebral segmentation. Therefore, this study aimed to discover variants associated with the NTV by considering the effect of the NV in pigs. The NTV and NV of 542 F2 individuals from a Large White $\times$ Minzhu pig crossbreed were recorded. All animals were genotyped for VRTN g.19034 A > C, LTBP2 c.4481A > C, and 37 missense or splice variants previously reported in a 951-kb interval on SSC7 and 147 single nucleotide polymorphisms (SNPs) on SSC14. To identify NTV-associated SNPs, we firstly performed a genome-wide association study (GWAS) using the $\mathrm{Q}+\mathrm{K}$ (population structure + kinship matrix) model in TASSEL. With the NV as a covariate, the obtained data were used to identify the SNPs with the most significant genome-wide association with the NTV by performing a GWAS on a PorcineSNP60K Genotyping BeadChip. Finally, a conditional GWAS was performed by fixing this SNP. The GWAS showed that 31 SNPs on SSC7 have significant genome-wide associations with the NTV. No missense or splice variants were found to be associated with the NTV significantly. A linkage disequilibrium analysis suggested the existence of quantitative trait loci (QTL) in a 479-Kb region on SSC7, which contained a critical candidate gene FOS for the NTV in pigs. Subsequently, a conditional GWAS was performed by fixing M1GA0010658, the most significant of these SNPs. Two SNPs in BMPR1A were found to have significant genome-wide associations and a significant dominant effect. The leading SNP, S14_87859370, accounted for $3.86 \%$ of the phenotypic variance. Our study uncovered that regulation variants in FOS on SSC7 and in BMPR1A on SSC14 might play important roles in controlling the NTV, and thus these genetic factors may be harnessed for increasing the NTV in pigs.
\end{abstract}


Keywords: BMPR1A; FOS; genome-wide association study; number of thoracic vertebrae; pig

\section{Introduction}

The mammalian spine is composed of the following five types of morphologically different vertebrae: cervical, thoracic, lumbar, sacral, and caudal. In pigs, the number of cervical vertebrae is fixed at seven, as in almost all mammals [1]. Both the number of thoracic vertebrae (NTV), which is equal to the number of ribs (NR), and the number of lumbar vertebrae are variable in pigs. The term "number of vertebrae" (NV) is generally used for referring to the total number of thoracic and lumbar vertebrae [2]. The NTV and NV in pigs vary, ranging between 13 and 16, and between 19 and 22, respectively [3]. In Chinese pork markets, ribs are among the most valuable parts of the animal. In addition, the higher the NTV is, the longer the animal [4]. Hence, increasing the NTV has been an important goal in pig husbandry. Recently, molecular approaches have increasingly been sought to generate pigs with a desired NTV since it is difficult to characterize the NTV in live animals for phenotypic selection.

The NTV in pigs is a highly heritable trait (heritability from 0.24 to 0.78 ) and shows considerable variation across breeds [3,5]. Many studies have shown that the NTV is a polygenic trait influenced by many different quantitative trait loci (QTL) on Sus scrofa Chromosomes (SSCs) 1, 2, 4, 7, 11, 16, and 18 [6-14]. Genome-wide association studies (GWASs) in recent years have shown that the major genes controlling the NTV in pigs are concentrated on SSC7 [15-17]. It is known that the total number of vertebrae is determined during development by the number of somites, developing from the presomitic mesoderm (PSM) via the Notch, FGF, Wnt, and Retinoic acid pathways. Concurrently, the vertebral pattern, which refers to the division of cervical, thoracic, lumbar, sacral, and caudal vertebrae along the embryo anterior-posterior axis, is resolved according to the expression patterns of Hox genes [18]. The genetic correlation coefficient between the NTV and NV ranged from 0.79 to 0.97 in pigs [19]. These findings suggest that number of thoracic or lumbar vertebrae are controlled by both somite segmentation and vertebral patterning. Although we have previously discovered similar QTLs for the NTV [16,20] as in other studies [6-14], our previous view on the NTV as an independent trait [20] seems incomplete because of insufficient consideration of the effect of the NV. Hence, the aim of this study was to discover genome-wide association variants for the NTV in pigs by considering the effect of the NV.

\section{Materials and Methods}

\subsection{Ethics Statement/Sample Description}

All animals were sacrificed by electric shock in Beijing Fifth Meat Processing Factory according to the guidelines for experimental animals established by the Council of China. The experiments involving animals were approved by the Science Research Department of the Institute of Animal Science, Chinese Academy of Agricultural Sciences (CAAS) (Beijing, China) (No. IASCAAS-PG-39).

\subsection{Phenotypic Data, Heritability, and Genetic Correlation between the NTV and NV}

The phenotypic data included the NTV and NV of 542 individuals from the F2 progeny of 4 Large White pigs crossed with 16 Minzhu pigs. The F1 generation consisted of 9 boars and 46 sows which were mated to produce $542 \mathrm{~F} 2$ animals $(65 \mathrm{~L})$ in three parities. All these F2 individuals were slaughtered at $240 \pm 7 \mathrm{~d}$ of age, and the phenotypes obtained from the carcasses were recorded. Genetic parameters pertaining to the NTV and NV were estimated using DMU software with pedigree-based linear models that are based on the following equation [21]:

$$
y=\mu+a+e
$$


where $y$ is the NTV or NV of the animal, $\mu$ is the overall mean, $a$ is the random additive genetic effect of the animal, and $e$ is the random residual effect.

2.3. Genotyping for VRTN g.19034 A>C 37, VRTN g.20311_20312ins291, LTBP2 c.4481A>C, and Missense or Splice Variants on SSC7 and 147 SNPS on SSC1

All the animals were genotyped for "Vertebrae Development Associated" (VRTN) g.19034 A>C, g.20311_20312ins291 [18] and "Latent Transforming Growth Factor Beta-binding Protein 2" (LTBP2) c.4481A > C [15]. Through comparison with the Sus scrofa genetic variant database in Ensembl (http://asia.ensembl.org/Sus_scrofa/Location/Variant), 37 missense or splice variants in a previously reported 951-kb interval on SSC7 [16] were obtained, and the animals were then genotyped for these variants as well. The information about the above 39 mutations and the primers used for genotyping are displayed in Supplementary Table S1. Additionally, a total of 147 SNPs (single nucleotide polymorphisms) on SSC14 were genotyped for each individual. The primers used for these variants were designed using Primer3 (http://primer3.ut.ee/) (see Supplementary Table S2). Genomic DNA from each individual was extracted and used as the template for touchdown polymerase chain reaction (PCR). Touchdown PCR was carried out in $25-\mu \mathrm{L}$ volumes containing $0.2 \mu \mathrm{M}$ of each primer, $1.5 \mathrm{mM}$ $\mathrm{MgCl}_{2}, 0.2 \mathrm{mM}$ of each deoxynucleotide triphosphate, $4 \mathrm{ng} / \mu \mathrm{L}$ genomic DNA, and $0.03 \mathrm{U} / \mu \mathrm{L}$ Taq DNA polymerase with $1 \times$ buffer (TaKaRa, Beijing, China) under the following conditions: $94{ }^{\circ} \mathrm{C}$ for $5 \mathrm{~min}$; 5 cycles at $60{ }^{\circ} \mathrm{C}$ for $20 \mathrm{~s}$ and $72{ }^{\circ} \mathrm{C}$ for $45 \mathrm{~s} ; 30$ cycles at $52{ }^{\circ} \mathrm{C}$ for $20 \mathrm{~s}$ and $72{ }^{\circ} \mathrm{C}$ for $45 \mathrm{~s} ; 72{ }^{\circ} \mathrm{C}$ for $10 \mathrm{~min}$. The PCR products were resolved using 1.5\% agarose-gel electrophoresis (Promega, Madison, WI, USA) and photographed under UV light. They were then purified and sequenced on an ABI 377 sequencer (ABI, Foster City, CA, USA).

\subsection{Genome-Wide Association Study Based on the SNPs and Variants That Merged with Porcine SNP60K Genotyping BeadChip Assays}

Population structure and cryptic relationships were considered to minimize false positives and increase statistical power. The kinship derived from the whole-genome SNPs was set as a random effect to control for family effects. The GWAS for the NTV was performed using the TASSEL 5.0 (Institute for Genomic Diversity, Cornell University, Ithaca, NY, USA) software [22] with the data from Porcine SNP60K BeadChip genotyping [20]. A sample call rate of $>90 \%$, an SNP call rate of $>90 \%$, a minor allele frequency of $>5 \%$, a maximum missing rate of $<0.9$, and only two alleles were used to evaluate data quality. A mixed linear model $(\mathrm{Q}+\mathrm{K})$ was applied in this GWAS. Principal component analysis (PCA) was performed. The kinship matrix was calculated using the default method (centered IBS (Identity by state)) [23] in TASSEL software. The " $Q$ " matrix was determined using the PCA which was run on the genotype data set to account for the effects resulting from the population structure, and the kinship matrix $(\mathrm{K})$ was calculated to replace pedigrees. The genotype at each SNP site and the first three PCAs were included as fixed effects, and a polygenic genetic effect was fitted as a random effect. The additive and dominant effects were estimated by the TASSEL software. The Bonferroni test whole-genome significance threshold was defined as $0.05 /$ total SNPs $\left(p=1.10 \times 10^{-6}\right)$. The phenotypic variation explained by each SNP is calculated based on a formula for $\mathrm{R}^{2}$ using Tassel software as shown here (https://bitbucket.org/tasseladmin/tassel-5-source/wiki/UserManual/MLM/ MLM).

$$
\mathrm{R}^{2}=\frac{\left(\hat{Y}_{\text {full }}-\hat{Y}_{\text {reduced }}\right)^{T} V^{-1}\left(\hat{Y}_{\text {full }}-\hat{Y}_{\text {reduced }}\right)}{(Y-\bar{Y})^{T} V^{-1}(Y-\bar{Y})}
$$

Since SNP M1GA0010658 showed the maximum association with the NTV, a conditional GWAS was re-run with this SNP fixed to detect novel associated variants. We calculated the distance between each significant genome-wide SNP and the annotated genes upstream and downstream of the SNP by using the Ensembl database (http://asia.ensembl.org). The nearest gene to the SNP was considered as a candidate gene for NTV. 


\subsection{Identification of the Effect of S14_87859370 on the NTV in Large White and Songliao Black Pigs}

A total of 214 Large White (LW) pigs from VICA group Co., Ltd. (Huludao, China), and 129 Songliao Black (SLB) pigs from the Jilin Academy of Agricultural Sciences were used in this study. The NTV was recorded after slaughter. Genotypic effects were analyzed using a one-way ANOVA through the GLM procedure of the SAS software package version 8.2 (SAS Institute, Inc., Cary, NC, USA) with genotype as a fixed effect. Duncan's multiple-range tests were used for assessing the significance of the differences among the means of different genotypes.

\subsection{Linkage Disequilibrium Analysis}

Linkage disequilibrium analysis was performed on the chromosomal region which contained all the SNPs significantly associated with the NTV on SSC7 and SSC14, respectively. The genotypes of all SNPs for 542 individuals and pedigrees were used to detect haplotype blocks. The visualized haplotype blocks were detected using the HAPLOVIEW V3.31 program [21] with default parameters.

\section{Results}

\subsection{Phenotypes and Genetic Parameters Pertaining to the NTV and NV}

The NTV and NV phenotypes observed are summarized in Table 1 . The NTV was found to be 14 , 15 , and 16 in 193, 308, and 41 individuals, respectively. Interestingly, there were no individuals with 14 thoracic vertebrae and 22 vertebrae or with 16 thoracic vertebrae and 20 vertebrae. Using DMU software, the heritability values of the NTV and NV were estimated to be 0.59 and 0.54 , respectively. The genetic correlation between the two traits reached 0.77 .

Table 1. The distribution of the number of thoracic vertebrae in different groups with different numbers of thoracolumbar vertebrae.

\begin{tabular}{ccccc}
\hline \multirow{4}{*}{ Traits } & & \multicolumn{3}{c}{ NTV } \\
\cline { 3 - 5 } & & $\mathbf{1 4}$ & $\mathbf{1 5}$ & $\mathbf{1 6}$ \\
\hline \multirow{3}{*}{ NV } & 20 & 146 & 88 & 0 \\
& 21 & 47 & 209 & 19 \\
& 22 & 0 & 11 & 22 \\
\hline
\end{tabular}

NTV, number of thoracic vertebrae. NV, number of vertebrae.

\subsection{GWAS with the NTV by Using the Merged SNP Data from 542 F2 Animals Revealed Significant Variants}

Using the merged SNP data obtained from the $542 \mathrm{~F} 2$ individuals, a GWAS revealed a strong association peak (QTL) on SSC7 (Figure 1). The Q-Q plot (Quantile-Quantile plot) is shown in Supplementary Figure S1A. A total of 31 SNPs in a 25.76-Mb region (from 80.26 to $106.03 \mathrm{Mb}$ ) on SSC7 showed significant genome-wide associations with the NTV (Table 2). The SNPs most significantly associated with the NTV corresponded to the variations of M1GA0010658 in "Placental Growth Factor" $(P G F)$, and these variations could explain $7.80 \%$ of the phenotypic variance. All 31 SNPs showed significant additive effects with no dominant effect. This region contained a previously reported 951-kb interval, which comprised the reported candidates VRTN and LTBP2 for the NTV. In addition, 37 non-synonymous variants were detected during the whole-genome sequencing, and each animal was genotyped for these variants. For VRTN g.20311_20312ins291, no polymorphism was detected in this population. To evaluate the effects of these variants, LTBP2 c.4481 A > C, and VRTN g.19034 $A>C$, we identified them through a GWAS. The results suggested that the effects of these variants and two genes on the NTV were not significantly associated with the trait at the genome-wide level (Table 3). However, there were 1 and 5 SNPs on SSC 12 and 14, respectively, that had chromosome-wide 
associations with the NTV (Supplementary Table S3). The candidate genes were "Carbonic Anhydrase 10" (CA10) on SSC12 and BMPR1A on SSC14.

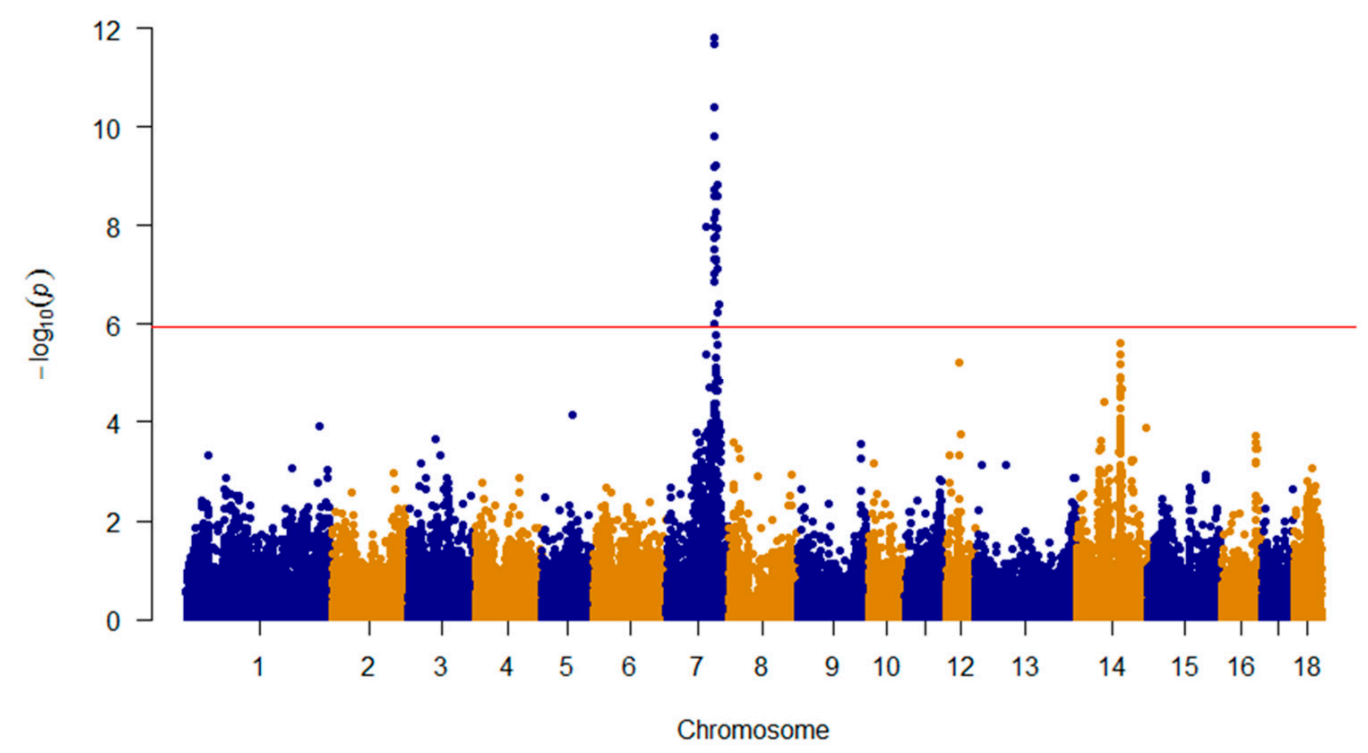

Figure 1. The Manhattan plot for genome-wide association studies for the NTV. The genome-wide association study (GWAS) was run on the NV treated as a covariate based on the 147 SNPs on SSC17 and 39 variants on SSC7 that merged with porcine SNP60K genotyping BeadChip assays. The red horizontal line indicated the Bonferroni significance threshold $\left(1.10 \times 10^{-6}\right)$.

\subsection{Linkage Disequilibrium Analysis Suggested Candidate Genes Are Contained in a 479-Kb Region on SSC7}

Within the 25.76-Mb region on SSC7, the linkage disequilibrium analysis detected four haplotype blocks as ALGA0043941-ALGA0043942 for $19 \mathrm{~kb}$, ALGA0043941-ALGA0043962 for 451 kb, DIAS0001088-ALGA0122954 for $479 \mathrm{~kb}$, and MARC0073299-DRGA0008061 for $469 \mathrm{~kb}$ (Figure 2). Since the 479-Kb block is the closest to the most significant SNP M1GA0010658, it could be regarded as the most important region harboring the candidate genes for the NTV. The 479-Kb region contained nine candidate genes: ribosomal protein 66 kinase like 1 (RPS6KL1), eukaryotic translation initiation factor 2B subunit beta (EIF2B2), mutL homolog 3 (MLH3), acylphosphatase 1 (ACYP1), NIMA related kinase 9 (NEK9), zinc finger C2HC-type containing 1C (ZC2HC1C), transmembrane p24 trafficking protein 10 (TMED10), Fos proto-oncogene, AP-1 transcription factor subunit (FOS), and Jun dimerization protein 2 (JDP2).

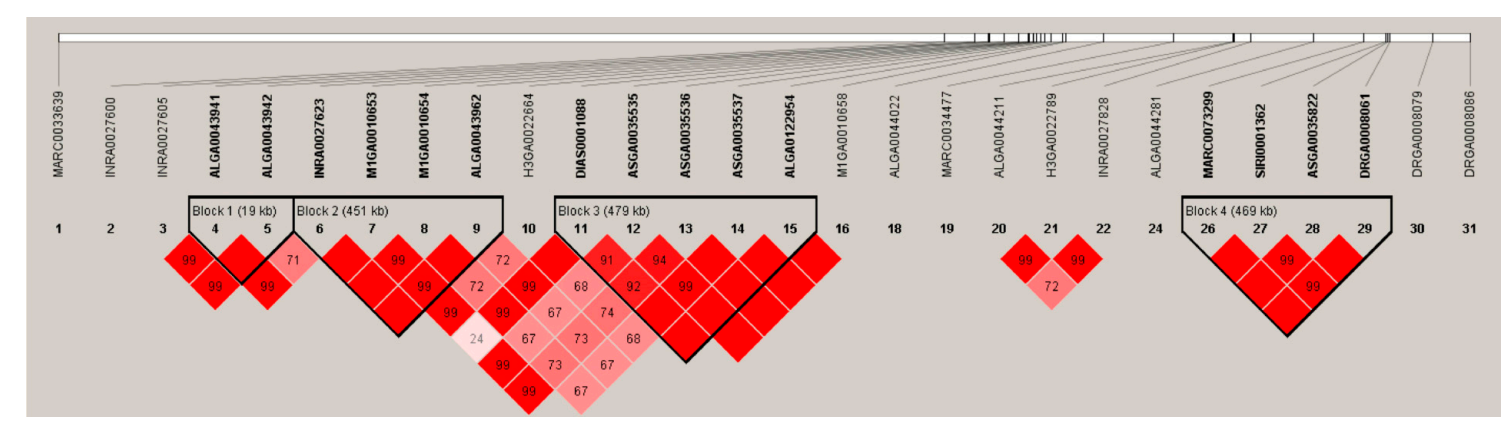

Figure 2. Haplotypes on a 25.76-Mb region on SSC7 containing all SNPs associated with the NTV significantly. The analysis was obtained using the HAPLOVIEW 3.31 program. A total of four blocks were identified and marked with solid lines. 
Table 2. Genome-wide association studies identified some associated variations for the NTV on SSC7 ${ }^{1}$.

\begin{tabular}{|c|c|c|c|c|c|c|c|c|}
\hline Marker & $\mathrm{Chr}^{2}$ & Position ${ }^{3}$ & $\mathrm{Rs}^{4}$ & $p$-Value & Add $\_p^{5}$ & Dom $\_p^{6}$ & Nearest Gene & $\operatorname{Var}(\%)^{7}$ \\
\hline MARC0033639 & 7 & 80264245 & rs80875505 & $1.06 \times 10^{-8}$ & $2.00 \times 10^{-12}$ & $1.02 \times 10^{-2}$ & SLC12A6 & 5.18 \\
\hline INRA0027600 & 7 & 96435072 & rs333341186 & $1.33 \times 10^{-7}$ & $2.38 \times 10^{-12}$ & $9.66 \times 10^{-3}$ & RBM25 & 4.44 \\
\hline INRA0027605 & 7 & 96994146 & rs337851273 & $9.12 \times 10^{-8}$ & $4.69 \times 10^{-12}$ & $3.78 \times 10^{-2}$ & ENSSSCG00000002348 & 4.55 \\
\hline ALGA0043941 & 7 & 97247184 & rs81396045 & $2.56 \times 10^{-9}$ & $2.05 \times 10^{-11}$ & $7.49 \times 10^{-2}$ & FAM161B & 5.59 \\
\hline ALGA0043942 & 7 & 97266221 & rs80856304 & $4.84 \times 10^{-8}$ & $7.41 \times 10^{-11}$ & $6.97 \times 10^{-3}$ & COQ6 & 4.74 \\
\hline INRA0027623 & 7 & 97521999 & rs321816080 & $1.03 \times 10^{-8}$ & $3.53 \times 10^{-10}$ & $1.14 \times 10^{-2}$ & $V S X 2$ & 5.18 \\
\hline M1GA0010653 & 7 & 97795697 & rs81396078 & $1.72 \times 10^{-8}$ & $4.32 \times 10^{-10}$ & $8.41 \times 10^{-4}$ & LTBP2 & 5.037 \\
\hline M1GA0010654 & 7 & 97954258 & rs80864705 & $1.87 \times 10^{-9}$ & $2.37 \times 10^{-10}$ & $8.79 \times 10^{-2}$ & FCF1 & 5.68 \\
\hline ALGA0043962 & 7 & 97973860 & rs80929215 & $1.87 \times 10^{-9}$ & $2.37 \times 10^{-10}$ & $8.79 \times 10^{-2}$ & $Y L P M 1$ & 5.68 \\
\hline H3GA0022664 & 7 & 98066911 & rs80813473 & $3.11 \times 10^{-8}$ & $8.24 \times 10^{-10}$ & $1.09 \times 10^{-3}$ & PROX2 & 4.863 \\
\hline DIAS0001088 & 7 & 98116120 & rs336641062 & $6.52 \times 10^{-10}$ & $3.30 \times 10^{-10}$ & $1.11 \times 10^{-1}$ & RPS6KL1 & 5.99 \\
\hline ASGA0035535 & 7 & 98186259 & rs80963494 & $1.49 \times 10^{-10}$ & $4.32 \times 10^{-9}$ & $1.45 \times 10^{-2}$ & EIF2B2 & 7.01 \\
\hline ASGA0035536 & 7 & 98264173 & rs 80846252 & $3.92 \times 10^{-11}$ & $9.72 \times 10^{-10}$ & $3.34 \times 10^{-2}$ & ACYP1 & 6.91 \\
\hline ASGA0035537 & 7 & 98374939 & rs 80854726 & $7.21 \times 10^{-9}$ & $4.42 \times 10^{-9}$ & $1.97 \times 10^{-1}$ & TMED10 & 5.30 \\
\hline ALGA0122954 & 7 & 98595714 & rs 81317665 & $2.03 \times 10^{-12}$ & $2.17 \times 10^{-9}$ & $7.21 \times 10^{-4}$ & $J D P 2$ & 7.74 \\
\hline M1GA0010658 & 7 & 98648325 & rs80804788 & $1.55 \times 10^{-12}$ & $9.66 \times 10^{-9}$ & $2.23 \times 10^{-2}$ & PGF & 7.80 \\
\hline ALGA0108658 & 7 & 98648325 & rs81336593 & $9.67 \times 10^{-7}$ & $4.26 \times 10^{-9}$ & $1.22 \times 10^{-2}$ & $J D P 2$ & 3.88 \\
\hline ALGA0044022 & 7 & 99337831 & rs80919617 & $5.78 \times 10^{-10}$ & $2.22 \times 10^{-9}$ & $4.40 \times 10^{-3}$ & GPATCH2L & 6.04 \\
\hline MARC0034477 & 7 & 100621452 & rs80954820 & $4.91 \times 10^{-8}$ & $2.16 \times 10^{-6}$ & $2.02 \times 10^{-1}$ & SPTLC2 & 4.74 \\
\hline ALGA0044211 & 7 & 101703098 & rs80950372 & $5.03 \times 10^{-8}$ & $1.01 \times 10^{-8}$ & $3.80 \times 10^{-3}$ & NRXN3 & 4.72 \\
\hline H3GA0022789 & 7 & 101728012 & rs80933409 & $5.52 \times 10^{-9}$ & $1.03 \times 10^{-8}$ & $6.25 \times 10^{-2}$ & NRXN3 & 5.37 \\
\hline INRA0027828 & 7 & 102031355 & rs323989598 & $1.60 \times 10^{-8}$ & $7.11 \times 10^{-9}$ & $2.05 \times 10^{-2}$ & $N A$ & 5.06 \\
\hline ASGA0035786 & 7 & 103002983 & rs80802872 & $2.46 \times 10^{-9}$ & $1.71 \times 10^{-8}$ & $6.72 \times 10^{-3}$ & DIO2 & 5.63 \\
\hline ALGA0044281 & 7 & 103164950 & rs 80808662 & $1.17 \times 10^{-8}$ & $1.15 \times 10^{-8}$ & $8.36 \times 10^{-3}$ & $N A$ & 5.15 \\
\hline H3GA0022821 & 7 & 103189827 & rs 80970878 & $1.45 \times 10^{-9}$ & $1.17 \times 10^{-8}$ & $8.56 \times 10^{-3}$ & DIO2 & 5.76 \\
\hline MARC0073299 & 7 & 104087830 & rs80786139 & $1.15 \times 10^{-8}$ & $1.17 \times 10^{-8}$ & $8.56 \times 10^{-3}$ & STON2 & 5.15 \\
\hline SIRI0001362 & 7 & 104480447 & rs320949387 & $7.60 \times 10^{-8}$ & $7.80 \times 10^{-7}$ & $7.64 \times 10^{-2}$ & ENSSSCG00000049060 & 4.60 \\
\hline ASGA0035822 & 7 & 104525604 & rs80936448 & $7.68 \times 10^{-8}$ & $4.22 \times 10^{-7}$ & $1.40 \times 10^{-1}$ & ENSSSCG00000049060 & 4.60 \\
\hline DRGA0008061 & 7 & 104557781 & rs80813073 & $7.68 \times 10^{-8}$ & $9.73 \times 10^{-6}$ & $4.92 \times 10^{-1}$ & ENSSSCG00000049060 & 4.60 \\
\hline DRGA0008079 & 7 & 105341213 & rs80962000 & $5.53 \times 10^{-7}$ & $8.99 \times 10^{-8}$ & $1.66 \times 10^{-2}$ & ENSSSCG00000047467 & 4.03 \\
\hline DRGA0008086 & 7 & 106025579 & rs81295294 & $3.95 \times 10^{-7}$ & $3.22 \times 10^{-7}$ & $1.99 \times 10^{-1}$ & ENSSSCG00000045920 & 4.13 \\
\hline
\end{tabular}

${ }^{1}$ NTV, number of thoracic vertebrae. ${ }^{2}$ Chromosome ${ }^{3}$ Data from Sus scrofa Build 11.1. ${ }^{4}$ rs, reference SNP. ${ }^{5}$ add_p, $p$-value for additive effect. ${ }^{6}$ dom_p, $p$-value for dominant effect.

7 Var (\%), phenotypic variation explained by the SNP.
. 
Table 3. Association of 37 missense or splice region variants, VRTN g.19034 A > C, and LTBP2 c.4481 A $>\mathrm{C}$ on SSC7.

\begin{tabular}{|c|c|c|c|c|c|}
\hline Marker & Chr ${ }^{1}$ & Position $^{2}$ & $\operatorname{Rs}^{3}$ & $\operatorname{Var} \%{ }^{4}$ & $p$-Value \\
\hline S7_97537758 & 7 & 97537758 & rs336742966 & 0.67 & $8.99 \times 10^{-2}$ \\
\hline VRTN g.19034 A > C & 7 & 97614602 & rs709317845 & 2.99 & $2.41 \times 10^{-5}$ \\
\hline S7_97622681 & 7 & 97622681 & rs787326242 & 1.31 & $1.01 \times 10^{-2}$ \\
\hline S7_97623045 & 7 & 97623045 & rs1108261998 & 1.42 & $6.57 \times 10^{-3}$ \\
\hline S7_97662010 & 7 & 97662010 & rs696186042 & 0.52 & $1.58 \times 10^{-1}$ \\
\hline S7_97662082 & 7 & 97662082 & rs345827854 & 2.02 & $9.68 \times 10^{-4}$ \\
\hline S7_97662535 & 7 & 97662535 & rs332888554 & 1.19 & $1.51 \times 10^{-2}$ \\
\hline S7_97750084 & 7 & 97750084 & rs322330509 & 2.91 & $3.91 \times 10^{-5}$ \\
\hline LTBP2 c.4481 A > C & 7 & 97751432 & rs322260921 & 2.95 & $2.83 \times 10^{-5}$ \\
\hline S7_97765472 & 7 & 97765472 & rs339379718 & 1.6 & $3.58 \times 10^{-3}$ \\
\hline S7_97771260 & 7 & 97771260 & rs337082599 & 1.69 & $2.92 \times 10^{-3}$ \\
\hline S7_97775923 & 7 & 97775923 & rs335686067 & 0.24 & $4.45 \times 10^{-1}$ \\
\hline S7_97777490 & 7 & 97777490 & rs331228271 & 0.49 & $1.88 \times 10^{-1}$ \\
\hline S7_97895559 & 7 & 97895559 & rs341911129 & 1.49 & $5.30 \times 10^{-3}$ \\
\hline S7_97899571 & 7 & 97899571 & rs325918746 & 1.21 & $1.24 \times 10^{-2}$ \\
\hline S7_97901617 & 7 & 97901617 & rs322374710 & 2.63 & $8.55 \times 10^{-5}$ \\
\hline S7_97901619 & 7 & 97901619 & rs331788516 & 2.63 & $8.55 \times 10^{-5}$ \\
\hline S7_98073512 & 7 & 98073512 & rs80930259 & 2.78 & $5.24 \times 10^{-5}$ \\
\hline S7_98073927 & 7 & 98073927 & rs329005836 & 0.97 & $3.36 \times 10^{-2}$ \\
\hline S7_98074140 & 7 & 98074140 & rs339766519 & 1.5 & $4.63 \times 10^{-3}$ \\
\hline S7_98074438 & 7 & 98074438 & rs322346679 & 2.73 & $8.41 \times 10^{-5}$ \\
\hline S7_98116877 & 7 & 98116877 & rs344681928 & 0.18 & $5.21 \times 10^{-1}$ \\
\hline S7_98130124 & 7 & 98130124 & rs323664885 & 0.03 & $9.07 \times 10^{-1}$ \\
\hline S7_98203930 & 7 & 98203930 & rs787271115 & 0.36 & $2.77 \times 10^{-1}$ \\
\hline S7_98219169 & 7 & 98219169 & rs323701300 & 0.1 & $7.04 \times 10^{-1}$ \\
\hline S7_98219967 & 7 & 98219967 & rs344167352 & 0.29 & $3.52 \times 10^{-1}$ \\
\hline S7_98242037 & 7 & 98242037 & rs338693270 & 0.11 & $6.67 \times 10^{-1}$ \\
\hline S7_98242461 & 7 & 98242461 & rs694346166 & 0.56 & $1.29 \times 10^{-1}$ \\
\hline S7_98242725 & 7 & 98242725 & rs340407061 & 0.92 & $3.68 \times 10^{-2}$ \\
\hline S7_98243724 & 7 & 98243724 & rs713439416 & 1.08 & $2.03 \times 10^{-2}$ \\
\hline S7_98244079 & 7 & 98244079 & rs333141847 & 0.19 & $5.08 \times 10^{-1}$ \\
\hline S7_98266495 & 7 & 98266495 & rs329334983 & 1.98 & $1.57 \times 10^{-4}$ \\
\hline S7_98266534 & 7 & 98266534 & rs324580288 & 1.76 & $3.68 \times 10^{-4}$ \\
\hline S7_98266749 & 7 & 98266749 & rs342214814 & 1.58 & $3.35 \times 10^{-3}$ \\
\hline S7_98266963 & 7 & 98266963 & rs693150674 & 1.72 & $1.98 \times 10^{-3}$ \\
\hline S7_98279107 & 7 & 98279107 & rs323090151 & 1.84 & $1.36 \times 10^{-3}$ \\
\hline S7_98300295 & 7 & 98300295 & rs341533265 & 0.96 & $3.05 \times 10^{-2}$ \\
\hline S7_98451235 & 7 & 98451235 & rs319445329 & 0.28 & $3.68 \times 10^{-1}$ \\
\hline S7_98451601 & 7 & 98451601 & rs80846787 & 0.43 & $2.25 \times 10^{-1}$ \\
\hline
\end{tabular}

${ }^{1}$ Chromosome. ${ }^{2}$ Data from Sus scrofa Build 11.1. ${ }^{3}$ Reference SNP. ${ }^{4}$ Phenotypic variation explained by the significant SNPs.

3.4. Conditional GWAS and Haplotype Block Analysis Revealed BMPR1A as a Novel Candidate Gene Affecting the NTV

A conditional GWAS was performed by fixing the strongest significant SNP, M1GA0010658, on SSC7. Only the SNPs S14_87859370 and S14_87859377, located on SSC14, were determined to be significantly associated with the NTV (Figure 3A,B). The two SNPs were found to have significant dominant effects with no additive effects. The Q-Q plot is shown in Supplementary Figure S1B. Both were located in BMPR1A. The leading SNP was S14_87859370, which resided in intron 4 of BMPR1A (Figure 3C) and accounted for 3.86\% of the phenotypic variance in the NTV (Table 4). 
A

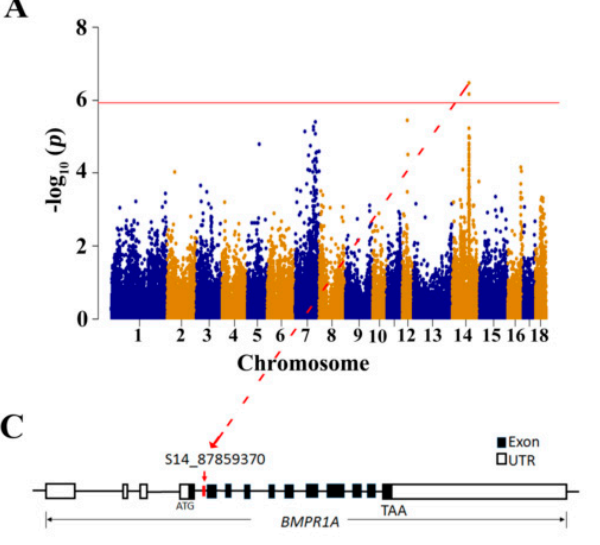

$\mathbf{E}$

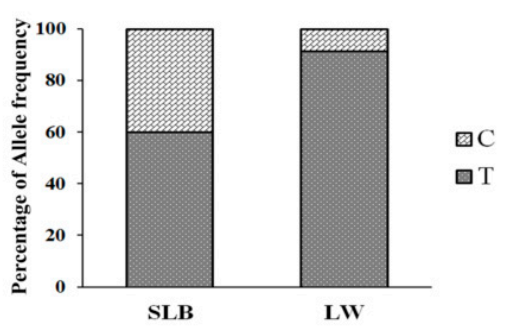

B

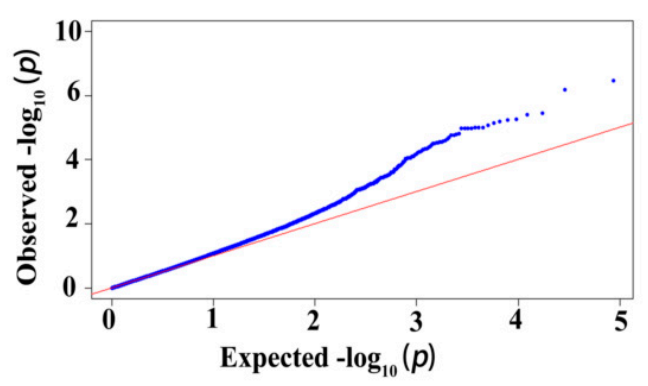

D

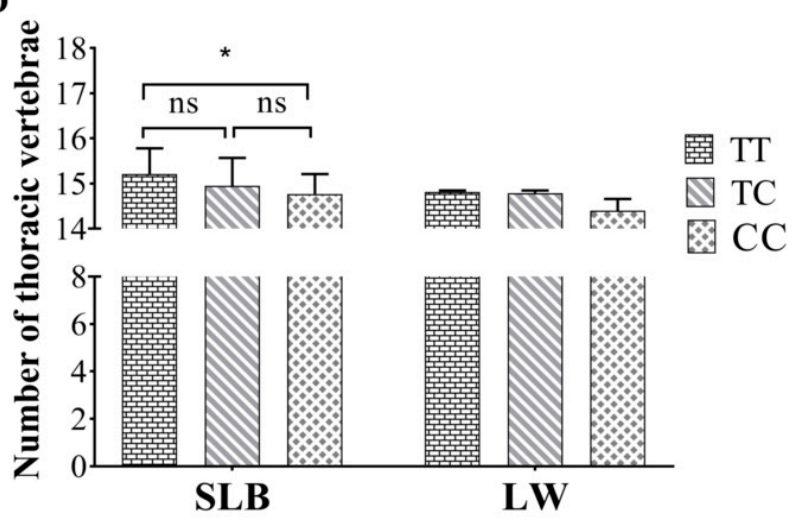

Figure 3. Identification of BMPR1A using conditional GWAS of the NTV. (A) Manhattan plot displaying the GWAS results of the NTV. The red horizontal line indicated the Bonferroni significance threshold $\left(1.10 \times 10^{-6}\right)$. Dots represent SNPs and the S14_87859370 is highlighted at the top dot. (B) The Q-Q plots obtained from the conditional GWAS for the NTV. (C) Red arrow represents the most significant SNP signal of the NTV. The S14_87859370 in the intron 4 of BMPR1A is marked in red. (D) The difference analysis among three genotypes of S14_87859370 for NTV in Songliao Black (SLB) and Large White (LW) pig populations. ${ }^{*}$ indicates $p<0.05$. (E) Allele frequencies of S14_87859370 in SLB and LW pig populations.

Table 4. Conditional genome-wide association study for the NTV ${ }^{1}$.

\begin{tabular}{ccccccccc}
\hline Marker & Chr $^{2}$ & Position $^{3}$ & $\boldsymbol{p}$-Value & Add_p & Dom_ $\boldsymbol{p}^{\mathbf{5}}$ & $\begin{array}{c}\text { Nearest } \\
\text { Gene }\end{array}$ & $\begin{array}{c}\text { Position } \\
\text { in Gene }\end{array}$ & Var (\%) $^{6}$ \\
\hline S14_87859370 & 14 & 87859370 & $3.40 \times 10^{-7}$ & $2.38 \times 10^{-3}$ & $6.71 \times 10^{-8}$ & $B M P R 1 A$ & Intron & 3.86 \\
S14_87859377 & 14 & 87859377 & $6.79 \times 10^{-7}$ & $2.28 \times 10^{-3}$ & $1.51 \times 10^{-7}$ & BMPR1A & Intron & 3.65 \\
\hline
\end{tabular}

${ }^{1}$ NTV, number of thoracic vertebrae. ${ }^{2}$ Chromosome. ${ }^{3}$ Data from Sus scrofa Build 11.1. ${ }^{4}$-value for additive effect.

${ }^{5} p$-value for dominant effect. ${ }^{6}$ Phenotypic variation explained by the significant SNPs.

To estimate the effect of S14_87859370 on the NTV, we genotyped pure lines of Songliao Black and Large White pigs for this SNP (Table 5). The Songliao Black pigs with the TT genotype had more NTV than those with the CC genotype $(p<0.05)$ (Figure 3D). Although the Large White pigs with the TT genotype had more thoracic vertebrae than those with the TC or CC genotype, the difference did not reach statistical significance since there were only two individuals with the CC genotype (Figure 3E). Nevertheless, in both lines, the frequency of the T allele was higher than that of the C allele (Figure 3E). 
Table 5. The association of S14_87859370 with the NTV in Songliao Black and Large White pigs ${ }^{1}$.

\begin{tabular}{cccc}
\hline Breed & Number & Genotype & Number of Thoracic Vertebrae $^{\mathbf{2}}$ \\
\hline \multirow{3}{*}{ Songliao Black } & 39 & TT & $15.21 \pm 0.57^{\mathrm{a}}$ \\
& 77 & TC & $14.95 \pm 0.62^{\mathrm{ab}}$ \\
& 13 & CC & $14.77 \pm 0.44^{\mathrm{b}}$ \\
\hline \multirow{3}{*}{ Large White } & 179 & TT & $14.82 \pm 0.03$ \\
& 33 & TC & $14.79 \pm 0.06$ \\
& 2 & CC & $14.40 \pm 0.26$
\end{tabular}

${ }^{1} \mathrm{NTV}$, number of thoracic vertebrae ${ }^{2}$ Completely different superscript letters indicate significant differences $(p<0.05)$.

\section{Discussion}

In this study, we did not observe any animals with an NTV of 14 or 16 in the populations with an NV of 22 or 20, respectively. The heritability of the NTV and NV was similar to that in previous reports [5]. The high genetic correlation (0.77) found in this study between the two traits indicates that the NTV and NV are closely related. Therefore, genetic analyses regarding the NTV should fully consider the effect of the NV. With the NTV considered an independent variable, only one QTL, located on SSC7, has been discovered $[16,17]$. However, when we considered the effect of the NV, our GWAS for the NTV detected 31 SNPs with genome-wide associations and located within a 22.93-Mb region (from 80.26 to $103.19 \mathrm{Mb}$ ) on SSC7, one chromosome-wide association SNP at 28.24 Mb on SSC12, and five chromosome-wide association SNPs within a 104.12-kb region (from 87.80 to $87.90 \mathrm{Mb}$ ) on SSC14. The 22.93-Mb region on SSC7 also included a previously reported 951-kb fragment consisting of important candidate genes-namely VRTN, LTBP2, and "Visual System Homeobox 2" (VSX2) [13,15-17,24-26]. Among these candidates, VRTN g.19034 A > C [17] and LTBP2 c.4481A > C [15] have been regarded as good candidate causal mutations. However, the association of either of them with the NTV did not reach genome-wide significance in our F2 population. Furthermore, a total of 37 non-synonymous variants addressed also displayed no significant association with the NTV in our GWAS.

On SSC7, a 479-Kb region including nine annotated genes was identified as a critical fragment influencing the NTV. Among these nine genes, FOS, also known as AP-1, is an important member of the Notch signaling pathway [27] and could be treated as the most important candidate gene for the NTV. The activation of FOS could inhibit the expression of Notch-1 [28], which is a critical gene for somite development [29]. That homozygous fos -/- mice displaying growth-retarded, develop osteopetrosis with deficiencies in bone remodeling and tooth eruption [30] suggested that this gene played an important role during bone development. Similar to a previous report [24], FOS was also regarded as a critical candidate gene for the porcine NTV in this work.

Surprisingly, when the effect of SSC7 was fixed, two variants on SSC14 showed significant genome-wide associations with the NTV. In addition, different from the SNPs on SSC7, we detected dominant effects at the two SNPs. To our knowledge, this is the first report with a dominant effect on NTV. These results suggested that the NTV was a complex trait with additive and dominant effects. Both of them were located in BMPR1A, which was selected as a good candidate due to its essential role in BMP (Bone morphogenetic protein) signaling [31]. BMPR1A, also known as ALK-3 and Brk-1, encodes a type I "Transforming Growth Factor- $\beta$ " (TGF- $\beta$ ) family receptor for the BMP-2 and BMP-4 signaling pathways [32]. In mice, BMP-2 is strongly expressed in the mid-somite region (anterior trunk of the embryo), and BMP-4 is robustly expressed in dorsal somites along the entire embryonic axis at E10.5 [33]. BMPs are secreted proteins that interact with cell-surface receptors to cause bone differentiation [34] and are believed to play various important roles during vertebrate embryogenesis [32]. The activation of the BMP signaling pathways requires the binding of BMPs to a hetero-oligomeric complex composed of type 1 and type 2 BMPRs. Three type 1 receptors (BMPR1a/Alk3, BMPR1b/Alk6, and ACVR1A/Alk2) and three type 2 receptors (BMPR2, ACTR2A, and ACTR2b) mediate most of the effects of BMPs [35]. 
Following the binding of BMPs to BMPR1/2 receptor heterodimers, receptor-regulated Smads (R-Smads) are activated. Subsequently, these activated R-Smads form hetero-oligomeric complexes with a common mediator Smad (co-Smad) and translocate into the nucleus, where they regulate the transcription of target genes [36,37]. Smads can modulate the transcriptional activities of Hox proteins, which act as general downstream DNA-binding proteins in the BMP/Smad signaling cascade [38]. Hox genes are key regulators of morphogenesis along the axial skeleton [39]. Vertebral patterning depends on ordered patterns of Hox gene expression as a mechanism for generating a combinatorial code that specifies the unique identities of the segments and their derivatives [40]. BMP signaling regulates vertebral specification when differentiating somites [41], and its perturbation in somitogenesis results in vertebral and rib malformations in the axial skeleton [42]. The discovery that mice lacking BMPR1A die by E9.5 due to defects in mesoderm formation [32] has led to the recognition of this protein as an essential factor for somitogenesis and vertebral development. Therefore, BMPR $1 A$ expression may control vertebral patterning via classical BMP-Smad-Hox signaling. These results indicate that S14_87859370, located in BMPR1A, is a potential binding site for a transcriptional regulator of the BMP pathway, and this observation may have important implications for further studies on the regulation of the NTV in pigs.

\section{Conclusions}

Our results suggest that regulation variants on SSC7 might play crucial roles affecting NTV. The FOS on SSC7 should be further studied as a critical candidate gene. On SSC14, to our knowledge, this is the first report showing that a natural variant in BMPR1A significantly contributes to the NTV in pigs. The identified variations provide valuable molecular information that may be harnessed to increase NTV in pigs.

Supplementary Materials: The following are available online at http://www.mdpi.com/2076-2615/10/11/2186/s1, Table S1: The information and primers for VRTN g.19034 A > C, LTBP2 c.4481A > C, and 37 missense or splice region variants in a reported 951-kb interval on SSC7, Table S2: The primers for 147 SNPs on SSC14, Table S3: Genome-wide association studies identified some chromosome-wide significant associated variations for the NTV, Figure S1: The Q-Q plots obtained from genome-wide association studies for the NTV.

Author Contributions: Conceptualization, L.Z. and L.W. (Lixian Wang); formal analysis, Q.L., J.Y., L.Z., and L.W. (Lixian Wang); investigation, X.L., Q.L., X.H., N.N., L.S., H.G., and H.Y.; methodology, Q.L., J.Y., F.Z., L.W. (Ligang Wang), and L.Z.; resources: L.W. (Ligang Wang) and L.Z.; supervision: X.L., L.Z., and L.W. (Lixian Wang); writing-original draft: L.Z. and Q.L.; writing—review and editing: L.Z. and L.W. (Lixian Wang). All authors have read and agreed to the published version of the manuscript.

Funding: This research was supported by the National Key Technology R\&D Program of China (2015BAD03B02-2), Beijing Natural Science Foundation (6174047), National Swine Industry Technology System (CARS-35), and Agricultural Science and Technology Innovation Program (ASTIP-IAS02).

Acknowledgments: We thank the members of Zhengkui Zhou (Institute of Animal Science, CAAS) and Bo Zuo (Huazhong Agricultural University) for helpful discussions. We are grateful to Lili Dong for uploading the sequence data to BIG Data Center.

Conflicts of Interest: The authors declare no conflict of interest.

\section{References}

1. Galis, F. Why do almost all mammals have seven cervical vertebrae? Developmental constraints, Hox genes, and cancer. Exp. Zool. 1999, 285, 19-26. [CrossRef]

2. Narita, Y.; Kuratani, S. Evolution of the vertebral formulae in mammals; a perspective on developmental constraints. Exp. Zool. B Mol. Dev. Evol. 2005, 304, 91-106. [CrossRef] [PubMed]

3. Zhang, Z.G.; Li, B.D.; Chen, X.H. Pig Breeds in China; Shanghai Scientific and Technical Publisher: Shanghai, China, 1986.

4. King, J.W.B.; Roberts, R.C. Carcass length in the bacon pig: Its association with vertebrae numbers and prediction from radiographs of the young pig. Anim. Prod. Sci. 1960, 2, 59-65. [CrossRef] 
5. Van Son, M.; Lopes, M.S.; Martell, H.J.; Derks, M.F.L.; Gangsei, L.E.; Kongsro, J.; Wass, M.N.; Grindflek, E.H.; Harlizius, B. A QTL for number of teats shows breed specific effects on number of vertebrae in pigs: Bridging the gap between molecular and quantitative genetics. Front. Genet. 2019, 10, 272. [CrossRef] [PubMed]

6. Zhang, Y.; Wang, M.; Yuan, J.; Zhou, X.; Xu, S.; Liu, B. Association of polymorphisms in NR6A1, PLAG1 and VRTN with the number of vertebrae in Chinese Tongcheng $\times$ Large White crossbred pigs. Anim. Genet. 2018, 49, 353-354. [CrossRef] [PubMed]

7. Zhang, J.; Xiong, Y.; Zuo, B.; Lei, M.; Jiang, S.; Li, F.; Zheng, R.; Li, J.; Xu, D. Detection of quantitative trait loci associated with several internal organ traits and teat number trait in a pig population. Genet. Genomics 2007, 34, 307-314. [CrossRef]

8. $\quad$ Edwards, D.B.; Ernst, C.W.; Raney, N.E.; Doumit, M.E.; Hoge, M.D.; Bates, R.O. Quantitative trait locus mapping in an F2 Duroc $\times$ Pietrain resource population: II. Carcass and meat quality traits. Anim. Sci. 2008, 86, 254-266. [CrossRef]

9. Choi, I.; Steibel, J.P.; Bates, R.O.; Raney, N.E.; Rumph, J.M.; Ernst, C.W. Identification of carcass and meat quality QTL in an $\mathrm{F}(2)$ Duroc $\times$ Pietrain pig resource population using different least-squares analysis models. Front. Genet. 2011, 2, 18. [CrossRef]

10. Casiró, S.; Velez-Irizarry, D.; Ernst, C.W.; Raney, N.E.; Bates, R.O.; Charles, M.G.; Steibel, J.P. Genome-wide association study in an $\mathrm{F}(2)$ Duroc $\times$ Pietrain resource population for economically important meat quality and carcass traits. Anim. Sci. 2017, 95, 545-558. [CrossRef]

11. Velez-Irizarry, D.; Casiro, S.; Daza, K.R.; Bates, R.O.; Raney, N.E.; Steibel, J.P.; Ernst, C.W. Genetic control of longissimus dorsi muscle gene expression variation and joint analysis with phenotypic quantitative trait loci in pigs. BMC Genomics 2019, 20, 3. [CrossRef]

12. Harmegnies, N.; Davin, F.; De Smet, S.; Buys, N.; Georges, M.; Coppieters, W. Results of a whole-genome quantitative trait locus scan for growth, carcass composition and meat quality in a porcine four-way cross. Anim. Genet. 2006, 37, 543-553. [CrossRef]

13. Mikawa, S.; Sato, S.; Nii, M.; Morozumi, T.; Yoshioka, G.; Imaeda, N.; Yamaguchi, T.; Awata, T. Identification of a second gene associated with variation in vertebral number in domestic pigs. BMC Genet. 2011, $12,5$. [CrossRef]

14. Yang, J.; Huang, L.; Yang, M.; Fan, Y.; Li, L.; Fang, S.; Deng, W.; Cui, L.; Zhang, Z.; Ai, H.; et al. Possible introgression of the VRTN mutation increasing vertebral number, carcass length and teat number from Chinese pigs into European pigs. Sci. Rep. 2016, 6, 19240. [CrossRef]

15. Park, H.B.; Han, S.H.; Lee, J.B.; Cho, I.C. Rapid Communication: High-resolution quantitative trait loci analysis identifies LTBP2 encoding latent transforming growth factor beta binding protein 2 associated with thoracic vertebrae number in a large F2 intercross between Landrace and Korean native pigs. Anim. Sci. 2017, 95, 1957-1962.

16. Zhang, L.C.; Yue, J.W.; Pu, L.; Wang, L.G.; Liu, X.; Liang, J.; Yan, H.; Zhao, K.B.; Li, N.; Shi, H.B.; et al. Genome-wide study refines the quantitative trait locus for number of ribs in a Large White $\times$ Minzhu intercross pig population and reveals a new candidate gene. Mol. Genet. Genom. 2016, 291, 1885-1890. [CrossRef]

17. Fan, Y.; Xing, Y.; Zhang, Z.; Ai, H.; Ouyang, Z.; Ouyang, J.; Yang, M.; Li, P.; Chen, Y.; Gao, J.; et al. A further look at porcine chromosome 7 reveals VRTN variants associated with vertebral number in Chinese and Western pigs. PLoS ONE 2013, 8, e62534. [CrossRef]

18. Gilbert, S.F. Developmental Biology, 6th ed.; Sinauer Associates: Sunderland, MA, USA, 2000.

19. Borchers, N.N.; Reinsch, N.; Kalm, E. The number of ribs and vertebrae in a Piétrain cross: Variation, heritability and effects on performance traits. Anim. Breed. Genet. 2004, 121, 392-403. [CrossRef]

20. Zhang, L.; Liu, X.; Liang, J.; Yan, H.; Zhao, K.; Li, N.; Pu, L.; Shi, H.; Zhang, Y.; Wang, L.; et al. Quantitative trait loci for the number of vertebrae on Sus scrofa chromosomes 1 and 7 independently influence the numbers of thoracic and lumbar vertebrae in pigs. J. Integrat. Agri. 2015, 14, 2027-2033. [CrossRef]

21. Madsen, P.; Jensen, J. DMU: A User's Guide. A Package for Analysing Ultivariate Mixed 253 Models. 2007, Version 6, Release 4.7. Available online: http://dmu.agrsci.dk/dmuv6_guide-R4-6-7.Pdf (accessed on 1 December 2014).

22. Bradbury, P.J.; Zhang, Z.; Kroon, D.E.; Casstevens, T.M.; Ramdoss, Y.; Buckler, E.S. TASSEL: Software for association mapping of complex traits in diverse samples. Bioinformatics 2007, 23, 2633-2635. [CrossRef] 
23. Endelman, J.B.; Jannink, J.L. Shrinkage estimation of the realized relationship matrix. G3 (Bethesda) 2012, 2, 1405-1413. [CrossRef]

24. Ren, D.R.; Ren, J.; Ruan, G.F.; Guo, Y.M.; Wu, L.H.; Yang, G.C.; Zhou, L.H.; Li, L.; Zhang, Z.Y.; Huang, L.S. Mapping and fine mapping of quantitative trait loci for the number of vertebrae in a White Duroc $\times$ Chinese Erhualian intercross resource population. Anim. Genet. 2012, 43, 545-551. [CrossRef]

25. Duan, Y.; Zhang, H.; Zhang, Z.; Gao, J.; Yang, J.; Wu, Z.; Fan, Y.; Xing, Y.; Li, L.; Xiao, S.; et al. VRTN is required for the development of thoracic vertebrae in mammals. Int. J. Biol. Sci. 2018, 14, 667-681. [CrossRef]

26. Guerreiro, I.; Casaca, A.; Nunes, A.; Monteiro, S.; Nóvoa, A.; Ferreira, R.B.; Bom, J.; Mallo, M. Regulatory role for a conserved motif adjacent to the homeodomain of Hox10 proteins. Development 2012, 139, 2703-2710. [CrossRef] [PubMed]

27. Chen, F.; Liu, N. A 10-gene expression signature of Notch pathway predicts recurrence in ovarian carcinoma. Oncol. Lett. 2015, 10, 1704-1708. [CrossRef]

28. Portanova, P.; Notaro, A.; Pellerito, O.; Sabella, S.; Giuliano, M.; Calvaruso, G. Notch inhibition restores TRAIL-mediated apoptosis via AP1-dependent upregulation of DR4 and DR5 TRAIL receptors in MDA-MB-231 breast cancer cells. Int. J. Oncol. 2013, 43, 121-130. [CrossRef]

29. Liao, B.K.; Oates, A.C. Delta-Notch signalling in segmentation. Arthropod Struct. Dev. 2017, 46, 429-447. [CrossRef]

30. Wang, Z.Q.; Ovitt, C.; Grigoriadis, A.E.; Möhle-Steinlein, U.; Rüther, U.; Wagner, E.F. Bone and haematopoietic defects in mice lacking c-fos. Nature 1992, 360, 741-745. [CrossRef]

31. Jing, J.; Hinton, R.J.; Feng, J.Q. Bmpr1a signaling in cartilage development and endochondral bone formation. Vitam. Horm. 2015, 99, 273-291.

32. Mishina, Y.; Suzuki, A.; Ueno, N.; Behringer, R.R. Bmpr encodes a type I bone morphogenetic protein receptor that is essential for gastrulation during mouse embryogenesis. Genes. Dev. 1995, 9, 3027-3037. [CrossRef]

33. Danesh, S.M.; Villasenor, A.; Chong, D.; Soukup, C.; Cleaver, O. BMP and BMP receptor expression during murine organogenesis. Gene Expr. Patterns 2009, 9, 255-265. [CrossRef]

34. Urist, M.R. Bone: Formation by autoinduction. Science 1965, 150, 893-899. [CrossRef]

35. Kishigami, S.; Mishina, Y. BMP signaling and early embryonic patterning. Cytokine Growth Factor Rev. 2005, 16, 265-278. [CrossRef]

36. Miyazono, K. Signal transduction by bone morphogenetic protein receptors: Functional roles of Smad proteins. Bone 1999, 25, 91-93. [CrossRef]

37. Matsumoto, Y.; Otsuka, F.; Hino, J.; Miyoshi, T.; Takano, M.; Miyazato, M.; Makino, H.; Kangawa, K. Bone morphogenetic protein-3b (BMP-3b) inhibits osteoblast differentiation via Smad2/3 pathway by counteracting Smad1/5/8 signaling. Mol. Cell. Endocrinol. 2012, 350, 78-86. [CrossRef]

38. Li, X.; Nie, S.; Chang, C.; Qiu, T.; Cao, X. Smads oppose Hox transcriptional activities. Exp. Cell Res. 2006, 312, 854-864. [CrossRef]

39. Wellik, D.M. Hox genes and vertebrate axial pattern. Curr. Top. Dev. Biol. 2009, 88, 257-278.

40. Alexander, T.; Nolte, C.; Krumlauf, R. Hox genes and segmentation of the hindbrain and axial skeleton. Annu. Rev. Cell Dev. Biol. 2009, 25, 431-456. [CrossRef]

41. Zhu, H.; Zhao, J.; Zhou, W.; Li, H.; Zhou, R.; Zhang, L.; Zhao, H.; Cao, J.; Zhu, X.; Hu, H.; et al. Ndrg2 regulates vertebral specification in differentiating somites. Dev. Biol. 2012, 369, 308-318. [CrossRef]

42. Nifuji, A.; Kellermann, O.; Kuboki, Y.; Wozney, J.M.; Noda, M. Perturbation of BMP signaling in somitogenesis resulted in vertebral and rib malformations in the axial skeletal formation. J. Bone Miner. Res. 1997, 12, 332-342. [CrossRef]

Publisher's Note: MDPI stays neutral with regard to jurisdictional claims in published maps and institutional affiliations.

(C) 2020 by the authors. Licensee MDPI, Basel, Switzerland. This article is an open access article distributed under the terms and conditions of the Creative Commons Attribution (CC BY) license (http://creativecommons.org/licenses/by/4.0/). 